\title{
Tuning the Mode Splitting of a Semiconductor Microcavity with Uniaxial Stress
}

\author{
Natasha Tomm $\odot,{ }^{1,}{ }^{*}$ Alexander R. Korsch $\odot,{ }^{2}$ Alisa Javadiø,${ }^{1}$ Daniel Najer $\odot,{ }^{1}$ Rüdiger Schott $\odot,{ }^{2}$ \\ Sascha R. Valentin $\odot,{ }^{2}$ Andreas D. Wieck $\odot,{ }^{2}$ Arne Ludwig $\odot,{ }^{2}$ and Richard J. Warburton ${ }^{1}$ \\ ${ }^{1}$ Department of Physics, University of Basel, Klingelbergstrasse 82, Basel CH-4056, Switzerland \\ ${ }^{2}$ Lehrstuhl für Angewandte Festkörperphysik, Ruhr-Universität Bochum, Bochum D-44780, Germany
}

(Received 18 February 2021; revised 20 April 2021; accepted 22 April 2021; published 27 May 2021)

\begin{abstract}
A splitting of the fundamental optical modes in micro- and nanocavities comprising semiconductor heterostructures is commonly observed. Given that this splitting plays a role in light-matter interaction and hence quantum technology applications, a method for controlling the mode splitting is useful. In this work we use an open microcavity composed of a "bottom" semiconductor distributed Bragg reflector (DBR) incorporating a $n-i-p$ heterostructure, paired with a "top" curved dielectric DBR. We measure the mode splitting as a function of wavelength across the stopband. We demonstrate a reversible in situ technique to tune the mode splitting by applying uniaxial stress to the semiconductor DBR. The method exploits the photoelastic effect of the semiconductor materials. We achieve a maximum tuning of approximately $11 \mathrm{GHz}$. The stress applied to the heterostructure is determined by observing the photoluminescence of quantum dots embedded in the sample, converting a spectral shift to a stress via deformation potentials. A thorough study of the mode splitting and its tuning across the stopband leads to a quantitative understanding of the mechanism behind the results.
\end{abstract}

DOI: 10.1103/PhysRevApplied.15.054061

\section{INTRODUCTION}

Semiconductor quantum dots (QDs) coupled to optical microcavities represent a useful platform to advance quantum-information technologies. Semiconductor QDcavity platforms, such as micropillars, photonic crystals, and open microcavities, have been successfully employed to achieve highly efficient single-photon sources $[1,2]$, a coherent light-matter interaction [3], generation of entangled photons [4,5], and photon-photon switches [6]. Despite the history of successful cavity quantumelectrodynamics demonstrations in these systems, there are still partly unresolved technical questions that affect their performance. One such property is the almost ubiquitous observation that the fundamental cavity mode splits into two separate modes with linear, orthogonal polarizations. This lifting of the polarization degeneracy is desired and exploited in some cases, notably in efficient single-photon sources in order to avoid a $50 \%$ loss of signal in crosspolarized collection schemes [1,2]. In this scenario, a QD

*natasha.tomm@unibas.ch

Published by the American Physical Society under the terms of the Creative Commons Attribution 4.0 International license. Further distribution of this work must maintain attribution to the author(s) and the published article's title, journal citation, and DOI. trion is excited via one cavity mode, and photons are preferentially emitted into the other cavity mode. In other cases however, polarization degenerate cavity modes are desirable. This is typically the case in experiments relying on circularly polarized excitation schemes [7], for instance a single spin in a perpendicular magnetic field. Here, the linearly polarized cavity modes result in a reduced coupling to the quantum emitter [8]. It is not simple to control the bare mode splitting precisely - it can depend on the local in-built strain in the material, and on fabrication imperfections. This suggests that a way of selectively tuning and controlling the mode splitting is of great interest: the bare mode splitting should be made large for a trion-based single-photon source; the bare mode splitting should ideally be eliminated for a single spin in an out-of-plane magnetic field. A tuning range in the range $10-50 \mathrm{GHz}$ is sufficient in many cases.

\section{BIREFRINGENCE INDUCED CAVITY MODE SPLITTING}

The polarization splitting of a semiconductor microcavity's fundamental mode is the result of birefringence in the semiconductor between two orthogonal crystalline axes (which are themselves orthogonal to the optical axis). In zinc-blende-type crystals there is a priori no intrinsic birefringence. Birefringence can be created however, often unintentionally, via two mechanisms. First, in heterostructures incorporating a diode or Schottky 
structure, the in-built electric field along the $z$ direction (growth axis) breaks the inversion symmetry of the crystal and birefringence in the $x-y$ plane arises via the linear electro-optic effect [9]. Secondly, a uniaxial stress in the $x-y$ plane, induced by microscopic imperfections in the heterostructure or postgrowth processing, induces birefringence via the photoelastic effect [10,11]. Contrarily, a biaxial stress does not result in observable birefringence on account of the symmetry of the zinc-blende crystal.

One can use the electro-optic and photoelastic effects to reverse or enhance the birefringence in semiconductor cavities, as previously demonstrated in monolithic structures [12-14]. Also in dielectric cavities it was demonstrated that the birefringence can be engineered to obtain a large degree of control over the cavity's mode splitting $[15,16]$. Here, we present a way of tuning the mode splitting of a hybrid dielectric-semiconductor open microcavity by making use of the photoelastic effect, i.e., the control of the birefringence upon application of uniaxial stress. A change in mode splitting of approximately $11 \mathrm{GHz}$ is achieved. Moreover, application of uniaxial stress to an open microcavity results in control not only of the mode splitting in the microcavity but also the absolute emission frequency of an embedded QD [17,18]. In this microcavity embodiment, the full stress is experienced by the entire heterostructure. This is not necessarily the case for monolithic systems.

\section{EXPERIMENTAL SETUP}

We employ a miniturized Fabry-Perot cavity $[2,3,19,20]$. The bottom mirror is a 46-pair $\operatorname{AlAs}(\lambda / 4) / \operatorname{GaAs}(\lambda / 4)$ semiconductor distributed Bragg reflector (DBR) grown on a [001] GaAs substrate, where $\lambda$ refers to the wavelength of light in the material. The bottom mirror's stopband is centered at $\lambda=918.7 \mathrm{~nm}$. The surface of the semiconductor heterostructure is passivated via an $\mathrm{Al}_{2} \mathrm{O}_{3}$ layer [21]. The top mirror is a 15pair $\mathrm{SiO}_{2}(\lambda / 4) / \mathrm{Ta}_{2} \mathrm{O}_{5}(\lambda / 4), \mathrm{Ta}_{2} \mathrm{O}_{5}$-terminated, dielectric DBR where the layers are deposited onto an approximately 600 -nm-deep microcrater with a radius of curvature approximately $15 \mu \mathrm{m}$ in a silica substrate, and its stopband is centered around $\lambda=930.0 \mathrm{~nm}$. The semiconductor heterostructure contains a layer of InAs QDs; the QDs themselves are embedded within a $n-i-p$ heterostructure, allowing the QD charge to be controlled via a voltage $\left(V_{g}\right)$ applied to the diode [2,3]. The sample is tightly glued onto a piezostack (PSt 150/7x7/7 cryo, Piezomechanik GmbH, Munich), as depicted in Fig. 1(a). The [1 10$]$ direction of the crystal aligns with the polarization axis of the pieozstack such that application of a voltage $V_{s}$ to the piezostack induces a $[1 \overline{1} 0]$ stress in the semiconductor. The spring constant of the sample is small compared to that of the piezostack, $k_{\text {sample }} \ll k_{\mathrm{PZT}}$, such that the extension of the piezo should be unaffected by the attached semiconductor. (a)

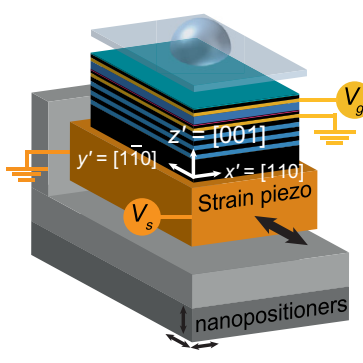

(b)

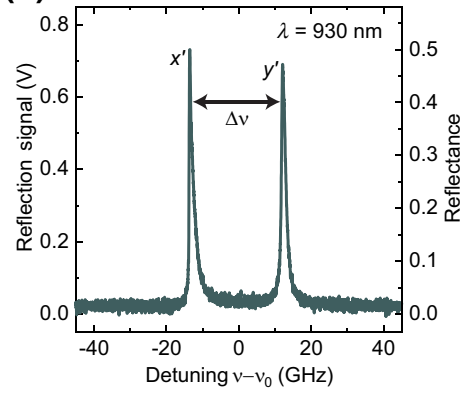

FIG. 1. (a) Experimental setup depicting the microcavity composed of the dielectric top mirror, and a semiconductor heterostructure, containing InAs QDs embedded in a $n-i-p$ diode structure (applied voltage $V_{g}$ ), and the bottom mirror. The sample is glued onto a piezostack (applied voltage $V_{S}$ ), that stresses the sample along the $y^{\prime}$ crystalline direction. The sample is positioned both laterally and vertically relative to the top mirror via nanopositioners. (b) Dark-field reflectivity scan across a cavity resonance: the fundamental mode is split into two linearly and orthogonally polarized modes. The microcavity axes are aligned with respect to the sample's crystalline axes $x^{\prime}$ and $y^{\prime}$. The $x^{\prime}$ polarized ( $y^{\prime}$-polarized) mode is red (blue) detuned from the expected resonance $v_{0}$. The orientation of the cavity modes is experimentally determined by aligning the polarization of the probing laser light to each cavity mode in turn, and by observing the alignment to the sample.

The piezosample assembly is mounted on a stack of $x-y-z$ nanopositioners, which are used to move it relative to the top mirror laterally, allowing different positions in the sample to be probed. The bottom mirror can also be moved vertically, changing the vacuum-gap separation between top and bottom mirrors, allowing a reflection spectrum of the microcavity to be recorded at fixed laser wavelength. We employ a cross-polarization confocal microscope [22], where an added half-wave plate (HWP) allows the probe laser's polarization to be aligned with one or the other polarized cavity mode. The sample's orientation relative to the microscope axes is known; the cleaved edges of the semiconductor sample along the $x^{\prime}=[110]$ and $y^{\prime}=[1 \overline{1} 0]$ crystalline axes coincide with the microscope orientation to within few degrees. All experiments are carried out at a temperature $T=4 \mathrm{~K}$.

The fundamental cavity mode is probed by measuring the reflectivity of a narrowband laser in a polarization darkfield modus. Two closely spaced modes are observed as shown in Fig. 1(b). When the HWP is set such that the probe laser's polarization is aligned to $x^{\prime}\left(y^{\prime}\right)$, only the red (blue) detuned resonance is probed. When the HWP is set such that the probe laser is aligned at $45^{\circ}$ to the $x^{\prime}$ and $y^{\prime}$ directions, both cavity modes can be seen [Fig. 1(b)]. These are the characteristic features of a birefringenceinduced mode splitting. The fact that the axes of the cavity modes are consistently aligned with the cleaved edges of 

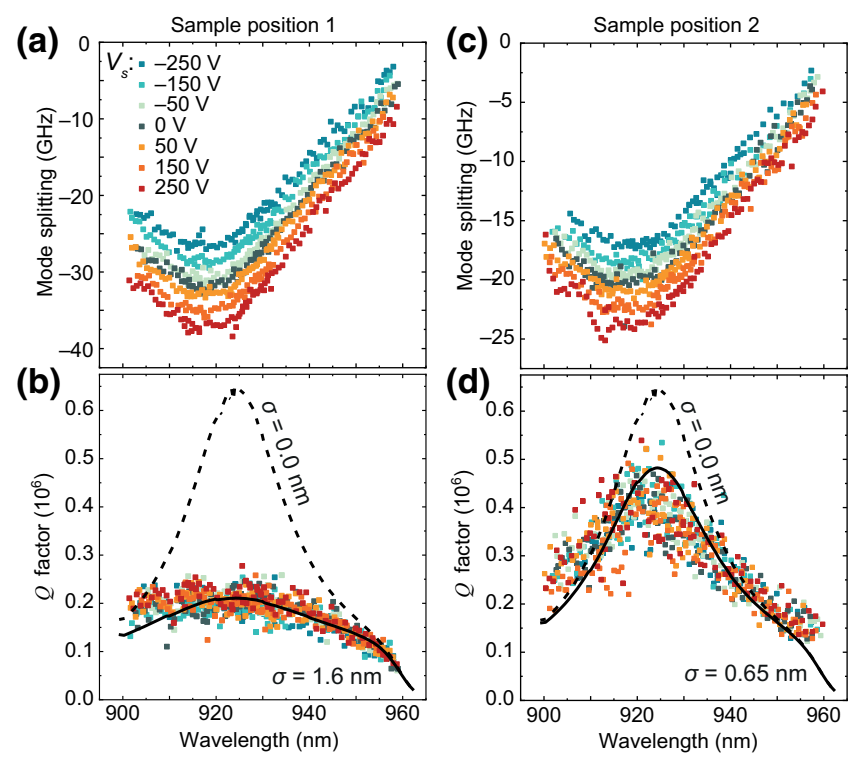

FIG. 2. Cavity mode splitting $\Delta v=v_{x^{\prime}}-v_{y^{\prime}}$ as a function of probe wavelength and voltage $V_{s}$ applied to the piezo at (a) position 1 , and (c) position 2 . The respective $\mathcal{Q}$ factors measured at these positions are shown in (b),(d). Plots (b),(d) show the modeled $\mathcal{Q}$-factor dispersion for this microcavity in the case without surface scattering $\sigma=0.0 \mathrm{~nm}$ at the semiconductorvacuum interface (dashed line), and with surface scattering. The rms surface roughness is $\sigma=1.60 \mathrm{~nm}$ (b) and $\sigma=0.65 \mathrm{~nm}$ (d) for the two positions evaluated (solid lines).

the sample implies that birefringence arises in the semiconductor heterostructure, and not in the top mirror. Should the origin of the birefringence lie in the top mirror, no link to the crystal axes of the semiconductor would be expected.

The mode splitting is defined by $\Delta v=v_{x^{\prime}}-v_{y^{\prime}}$, where $v$ is the resonance frequency. It is worthwhile to note that the mode splitting has a sign, negative in our case, meaning that the changes in refractive index along the $x^{\prime}$ and $y^{\prime}$ directions induce a red- and blueshift, respectively, relative to the original resonance. The dynamic nature of the microcavity allows us to examine simultaneously the mode splitting [Figs. 2(a) and 2(c)] and the $\mathcal{Q}$ factor across the microcavity's stopband [Figs. 2(b) and 2(d)]. Both the bare mode splitting and the $\mathcal{Q}$ factor have a dependence on wavelength with maximum amplitude centered around $\lambda=918.7 \mathrm{~nm}$, at the stopband center of the bottom mirror.

\section{CAVITY CHARACTERIZATION AND MODELING}

We focus initially on the $\mathcal{Q}$ factors to demonstrate that we have a quantitative understanding of both the field confinement in the microcavity and the losses. We model the microcavity's stopband and $\mathcal{Q}$-factor dependence on wavelength [Figs. 2(b) and 2(d) dashed and solid lines] using a one-dimensional transfer-matrix simulation (Essential Macleod, Thin Film Center Inc.). In Figs. 2(b) and 2(d) the dashed lines depict the expected $\mathcal{Q}$ factor without any losses at the sample's surface. In practice, the measured $\mathcal{Q}$ factors are lower and this can be described very convincingly simply by including the effects of scattering at the $\mathrm{Al}_{2} \mathrm{O}_{3}$-vacuum interface [21]. The surface roughness is determined by comparing the experimental results and the theoretical model. We find that the maximum $\mathcal{Q}$ factor in this experiment depends on the exact lateral position, suggesting that the surface roughness changes across the sample [21]. A full wavelength dependence was acquired at two positions on the sample. A rms surface roughness of $\sigma=1.60 \mathrm{~nm}(\sigma=0.65 \mathrm{~nm})$ at position 1 (position 2) provide a very good description of the wavelength dependence of the $\mathcal{Q}$ factor. These surface roughnesses are consistent with characterization of the surface at room temperature with atomic force microscopy [21]. The residual small discrepancy between experimental and modeled curves probably arises from an imperfect knowledge of the exact layer thicknesses in the DBRs.

\section{PHOTOELASTIC EFFECT}

We turn now to the behavior on applying a uniaxial stress. We focus on position 1. Upon application of a voltage up to $V_{s}= \pm 250 \mathrm{~V}$, the piezostack expands and contracts, thereby stressing the sample uniaxially along the $y^{\prime}$ direction. The mode splitting responds to the applied stress. A maximum mode splitting tuning of approximately 11 $\mathrm{GHz}(45 \mu \mathrm{eV})$ is achieved at the exact wavelength where $\left|v_{x^{\prime}}-v_{y^{\prime}}\right|$ is the largest, as can be observed in Fig. 2(a). The tuning leaves the $\mathcal{Q}$ factor unaltered [Fig. 2(b)] indicating that the applied stress has no effect on the loss mechanisms in these high- $\mathcal{Q}$-factor cavities. The mode splitting $\Delta v$ is a linear function of $V_{s}$ [Fig. 3(b)]; the response $\Delta v / \Delta V_{s}$ is slightly smaller in magnitude at the edges of the bottom mirror's stopband with respect to its stopband center [Fig. 3(d)].

We now attempt to understand quantitatively the stressinduced change in mode splitting. A crucial step is to determine the exact uniaxial stress applied. The extension per volt of the piezostack depends strongly on temperature and unfortunately we do not know its exact value at $T=4 \mathrm{~K}$. We do not have an external stress gauge in the experiment. Instead, we determine the applied stress by measuring the frequency shift of the photoluminescence from the QDs embedded in the sample [17]. This has the advantage of determining the stress experienced by the heterostructure itself, exactly the stress, which induces the birefringence. We determine the mean band-gap shift as a function of applied voltage $V_{s}$ by observing the photoluminescence signal of 20 different excitonic lines in ten QDs in the sample, as depicted in Fig. 4(a), and find $\Delta E_{\text {gap }} / \Delta V_{s}=$ $(-0.51 \pm 0.01) \mu \mathrm{eV} / \mathrm{V}$ equivalently $(-0.123 \pm 0.002)$ $\mathrm{GHz} / \mathrm{V}$ [Fig. 4(b)], a value comparable to a previously achieved [17] tuning of $-0.82 \mu \mathrm{eV} / \mathrm{V}$. The dominant 
effect of an applied differential uniaxial stress $\delta \sigma$ on the emission frequency of the QDs is to induce a change in the band gap $\delta E_{\text {gap }}$ of the host semiconductor GaAs [23-25], described by $\delta E_{\text {gap }} / \delta \sigma$. The influence of uniaxial stress on the band gap can be derived from the material's deformation potentials to be $\delta E_{\text {gap }} / \delta \sigma=-22.2 \mu \mathrm{eV} / \mathrm{MPa}$, under the assumption that the valence state is pure heavy hole. A detailed calculation is presented in the appendix. Finally, from

$$
\Delta \sigma / \Delta V_{s}=\frac{\delta E_{\text {gap }} / \delta V_{s}}{\delta E_{\text {gap }} / \delta \sigma}
$$

we infer $\left(\Delta \sigma / \Delta V_{s}\right)=(22.97 \pm 0.45) \mathrm{kPa} / \mathrm{V}$, from which we are able to deduce the amount of stress applied to the sample $\sigma=\left(\Delta \sigma / \Delta V_{s}\right) V_{s}$.

The next step is to calculate the birefringence in each layer in the heterostructure. Stress-induced transformations to the dielectric function of a crystal are quantified
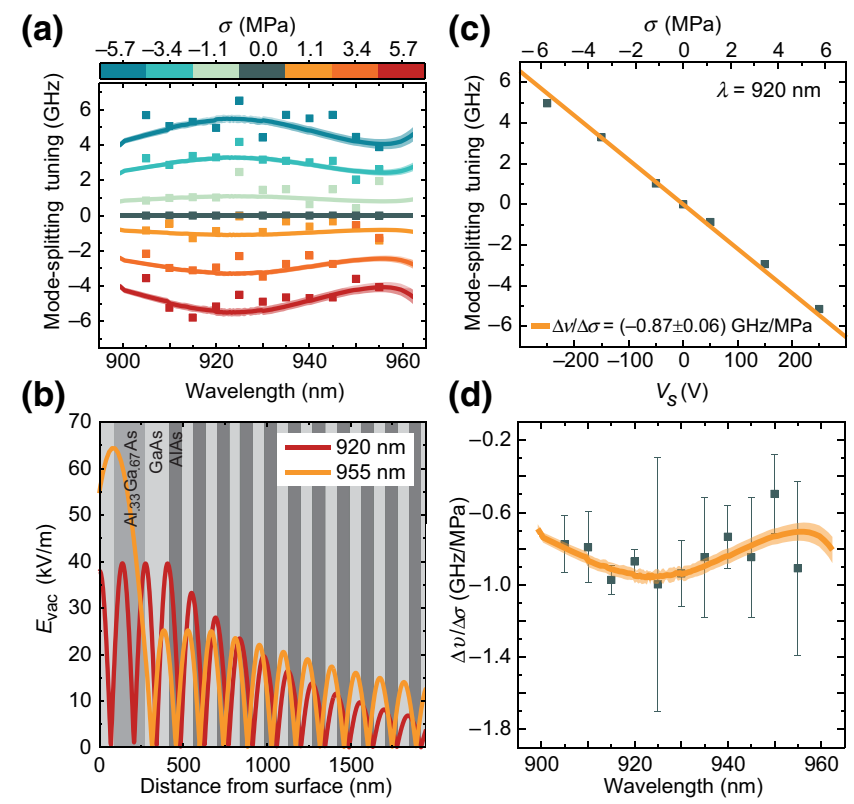

FIG. 3. (a) Change in mode splitting as a function of wavelength and applied uniaxial stress $\sigma$ from experimental data (dots) and theoretical model (solid lines). The model involves adjusting the refractive index of each layer in the heterostructure using Eqs. (1) and (2) and then calculating the exact resonance frequency in a one-dimensional transfer-matrix simulation. The error bars in the model arise from uncertainties in the coefficients $q_{44}$ and in the calculation of $\delta E_{\text {gap }} / \delta V_{s}$. (b) Vacuum electric field distribution along the first few hundred nanometers below the sample's surface at wavelengths of $920 \mathrm{~nm}$ and $955 \mathrm{~nm}$, indicating the dispersive influence of each layer's birefringence on the mode splitting. (c) The mode-splitting tuning at $\lambda=920 \mathrm{~nm}$ as a function of applied stress voltage $V_{s}$ (and respective stress $\sigma$ ). A linear fit determines the tuning rate $\Delta v / \Delta \sigma=(-0.87 \pm 0.06)$ $\mathrm{GHz} / \mathrm{MPa}$ at this wavelength. (d) The tuning rate $\Delta v / \Delta \sigma$ as a function of wavelength across the entire stopband: experiment (black symbols); model (orange line). by the so-called piezobirefringent tensor $[11,25-27] q_{i j k l}$. Due to the symmetry of zinc-blende crystals $[11,26]$, and our system of coordinates $x^{\prime}=[110], y^{\prime}=[1 \overline{1} 0], z^{\prime}=z=$ [001], the induced birefringence $\Delta n / n_{0}=\left(n_{x^{\prime}}-n_{y^{\prime}}\right) / n_{0}$ on stressing a semiconductor along $x^{\prime}$ by an amount $\sigma$ is given by

$$
\frac{\Delta n}{n_{0}}=-\frac{n_{0}^{2}}{2} \times q_{44} \times \sigma,
$$

where $n_{0}$ is the bare refractive index of the particular material, and $q_{44}$ is a material parameter, $q_{44}=p_{44} \times S_{44}$, where $p_{44}$ is an element of the photoelastic tensor and $S_{44}$ an element of the compliance tensor. See the appendix for complete derivation.

Given that the sample is composed of layers of three different semiconductor materials (GaAs, $\mathrm{Al}_{0.33} \mathrm{Ga}_{0.67} \mathrm{As}$, and $\mathrm{AlAs}$ ), the influence of uniaxial stress in each layer must be considered. The coefficients $q_{44}$ for GaAs, $\mathrm{Al}_{0.33} \mathrm{Ga}_{0.67} \mathrm{As}$, and $\mathrm{AlAs}$ at low temperature $T=4 \mathrm{~K}$ are estimated (see the appendix for details) from literature room-temperature values [28] and found to be $q_{44}=(-7.4 \pm 1.2) \times 10^{-13} \mathrm{~m}^{2} / \mathrm{N}, q_{44}=(-7.9 \pm 0.3) \times$ $10^{-13} \mathrm{~m}^{2} / \mathrm{N}$, and $q_{44}=(-1.64 \pm 0.02) \times 10^{-13} \mathrm{~m}^{2} / \mathrm{N}$, respectively.

Finally, we determine the mode splitting by calculating the exact mode frequency for each polarization separately, including the subtle changes to the refractive indexes in the one-dimensional transfer-matrix simulation. Specifically, we use Eq. (2) to calculate the induced birefringence $\Delta n$ in each layer of the heterostructure upon application of uniaxial stress $\sigma$, which is itself calculated with Eq. (1). For $V_{s}=250 \mathrm{~V}, \sigma=5.74 \mathrm{MPa}$, the induced relative birefringence $\Delta n / n_{0}$ is as small as $26 \mathrm{ppm}$ in GaAs (25 ppm in $\mathrm{Al}_{0.33} \mathrm{Ga}_{0.67} \mathrm{As}, 4 \mathrm{ppm}$ in AlAs). The stress tuning of the mode splitting is shown in Fig. 3(a) (solid lines) for each applied stress voltage $V_{s}$ as a function of wavelength (spanning the stopband). The results can be directly compared to the experimental results (symbols). Evidently
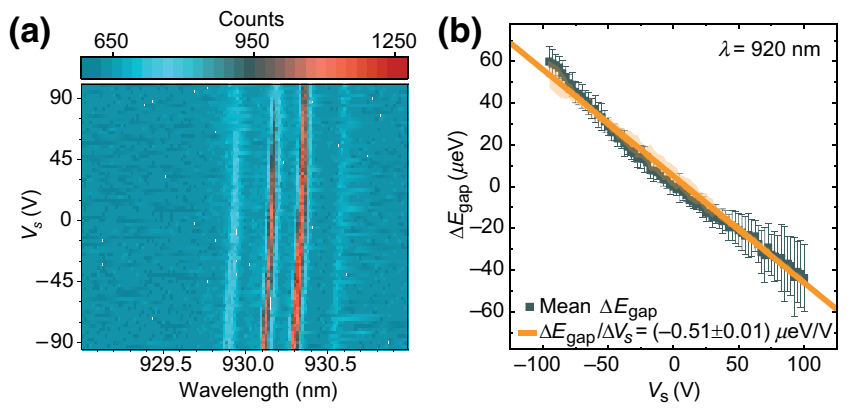

FIG. 4. (a) Shift of the photoluminescence signal of a QD in the sample as a function of applied uniaxial stress. (b) Mean shift in band-gap energy as a function of voltage applied to the piezostack $\left(V_{s}\right)$, as measured on 20 different excitonic lines in ten QDs. A linear fit gives $\delta E_{\text {gap }} / \delta V_{s}=(-0.51 \pm 0.01) \mu \mathrm{eV} / \mathrm{V}$. 
from Fig. 3(a), the amount of tuning itself presents a dispersion, i.e., it depends on wavelength. The calculation captures this detail precisely and explains it: subtle shifts in the standing wave in the microcavity change the net birefringence as each layer of the heterostructure does not contribute equally. Figure 3(b) illustrates this point by showing the vacuum electric field as a function of distance from the sample's surface at a wavelength close to the bottom mirror's stopband center, at $920 \mathrm{~nm}$, and at a wavelength far away, at $955 \mathrm{~nm}$. As a consequence, the mode splitting tunes linearly with stress, as depicted in Fig. 3(c) for $\lambda=920 \mathrm{~nm}$, but with different slopes $\Delta v / \Delta \sigma$ across the stopband [Fig. 3(d)]. Across the entire spectral range examined here, experimental data (points) and model (solid lines) present excellent agreement.

\section{CONCLUSIONS AND OUTLOOK}

Our method proves to be an effective way of controlling the intrinsic polarization splitting of an open semiconductor microcavity by up to $\pm 5.5 \mathrm{GHz}$. The mode splitting can be tuned across the entire stopband in a predictable, reversible manner. The present microcavity has a rather large intrinsic mode splitting. Nevertheless, the tuning capability allows us to achieve near degeneracy of the cavity modes at the high-wavelength end of the stopband. For a microcavity with a lower intrinsic mode splitting, it should be possible to eliminate the mode splitting. Of relevance here is the fact that the intrinsic mode splitting and the applied stress are aligned along the same axes. The applied stress induces a small birefringence, on the order of a few ppm, and does not influence the microcavity's $\mathcal{Q}$ factor. The slight emission shift of the QDs embedded in the heterostructure can be compensated for in the present setup simply by exploiting the spectral tunability of the microcavity: a resonance with the cavity mode is easily maintained.

Naturally, it is desirable to achieve a higher degree of mode-splitting tunability: perfect degeneracy of the polarized modes would be desirable for any experiments relying on circularly polarized transitions [7,8]; a further increase in the mode splitting until the two cavity modes overlap only weakly in frequency is sought in experiments that hinge on the vacuum field of just one of the cavity modes $[1,2]$. An optimized architecture of the sample holder could increase the tuning rate [18] by a factor of 20 . The incorporation of a back gate would allow an electric field to be applied across the bottom mirror, thereby making use of the electro-optic effect [13]. Employing these two methods simultaneously would grant an even higher degree of control of the birefringence. Additionally, inspired by liquid-crystal tuning of microcavities at room temperature [16], it might be possible to include a thin layer of a highly piezobirefringent layer in the present device. As a final comment, we note that while a quantitative understanding of the origin of the intrinsic mode splitting has not yet been attained, the mode-splitting dispersion curve can be used as a diagnostic tool as it indicates in which layers of the heterostructure birefringence is strong.

\section{ACKNOWLEDGMENTS}

We thank Liang Zhai for helpful advice on the experimental setup. We acknowledge financial support from SNF Project 200020_175748, NCCR QSIT and Horizon-2020 FET-Open Project QLUSTER. A.J. acknowledges support from the European Unions Horizon 2020 Research and Innovation Programme under the Marie SkłodowskaCurie Grant Agreement No. 840453 (HiFig). S.R.V., R.S., A.L., and A.D.W. acknowledge gratefully support from DFH/UFA CDFA05-06, DFG TRR160, DFG Project 383065199, and BMBF Q.Link.X No. 16KIS0867.

\section{APPENDIX}

\section{Photoelastic effect: the effect of uniaxial stress}

The refractive index $n$ of a crystal can be described by the indicatrix [26], an ellipsoid in which the principal axes represent the components of the dielectric tensor,

$$
\overline{\overline{B_{i j}}}=\varepsilon_{0} \frac{\partial E_{i}}{\partial D_{j}}=\left(\frac{1}{n^{2}}\right),
$$

where $\varepsilon_{0}$ is the vacuum's electric permittivity, $E_{i}$ is the electric field component along direction $i$ and $D_{j}$ is the electric displacement field along $j$. An applied stress deforms the indicatrix components $\Delta \overline{\overline{B_{i j}}}$ via the operation

$$
\Delta \overline{\overline{B_{i j}}}=\overline{\overline{q_{i j k l}}} \times \overline{\overline{\sigma_{k l}}}
$$

Here, $\overline{\overline{q_{i j k l}}}(i, j, k, l=1,2,3)$ is the fourth-rank piezobirefringent tensor; the stress $\overline{\overline{\sigma_{k l}}}(k, l=1,2,3)$ is a secondrank tensor. From Eqs. (A1) and (A2), it follows that the change in refractive index $\Delta \overline{\overline{n_{i j}}}=\overline{\overline{n_{i j}}}-n_{0}$ (where $n_{0}$ is the bare refractive index of the isotropic material) reads

$$
\Delta \overline{\overline{n_{i j}}}=-\frac{\Delta \overline{\overline{B_{i j}}}}{2} \times n_{0}^{3} .
$$

For zinc-blende-type (cubic) crystals, symmetry simplifies the photoelastic tensor such that only three independent coefficients remain [11], namely $q_{1111}, q_{1122}$, and $q_{2323}$. A compressed notation can be adopted: $11 \rightarrow$ $1,22 \rightarrow 2,33 \rightarrow 3,23 \rightarrow 4,13 \rightarrow 5,12 \rightarrow 6$. In this way, the rank of the tensors is reduced and the expression in Eq. (A2) becomes $\Delta \overline{B_{m}}=\overline{\overline{q_{m n}}} \times \overline{\sigma_{n}} \quad(m, n=$ 
$1,2,3,4,5,6)$. In extended form,

$$
\left[\begin{array}{l}
\Delta B_{1} \\
\Delta B_{2} \\
\Delta B_{3} \\
\Delta B_{4} \\
\Delta B_{5} \\
\Delta B_{6}
\end{array}\right]=\left[\begin{array}{cccccc}
q_{11} & q_{12} & q_{12} & 0 & 0 & 0 \\
q_{12} & q_{11} & q_{12} & 0 & 0 & 0 \\
q_{12} & q_{12} & q_{11} & 0 & 0 & 0 \\
0 & 0 & 0 & q_{44} & 0 & 0 \\
0 & 0 & 0 & 0 & q_{44} & 0 \\
0 & 0 & 0 & 0 & 0 & q_{44}
\end{array}\right]\left[\begin{array}{c}
\sigma_{1} \\
\sigma_{2} \\
\sigma_{3} \\
\sigma_{4} \\
\sigma_{5} \\
\sigma_{6}
\end{array}\right] .
$$

We now apply these general results to our problem. In the experiment, both the stress and the birefringence are applied and probed in the $\left(x^{\prime}, y^{\prime}, z^{\prime}\right)$ system of coordinates. Therefore, a rotation in the frame of reference by $\pi / 4$ around $z=z^{\prime}$ is applied. We treat the canonical case of a stress applied along the $x^{\prime}$ direction.

In the $\left(x^{\prime}, y^{\prime}, z^{\prime}\right)$ basis, the simplified stress tensor for a uniaxial stress along $x^{\prime}$ is self-evidently $\overline{\sigma^{\prime}}=\left[\begin{array}{lllll}1 & 0 & 0 & 0 & 0\end{array}\right]^{\top}$. We start by calculating $\bar{\sigma}$ in the usual basis $(x, y, z)$ from $\overline{\sigma^{\prime}}$. The general rotation matrix for an arbitrary angle $\theta$ and with $\theta=\pi / 4$ is

$$
\overline{\bar{R}}=\left[\begin{array}{rrr}
\cos \theta & \sin \theta & 0 \\
-\sin \theta & \cos \theta & 0 \\
0 & 0 & 1
\end{array}\right] \stackrel{\theta=\pi / 4}{=}\left[\begin{array}{ccc}
\frac{1}{\sqrt{2}} & \frac{1}{\sqrt{2}} & 0 \\
\frac{-1}{\sqrt{2}} & \frac{1}{\sqrt{2}} & 0 \\
0 & 0 & 1
\end{array}\right]
$$

In the $(x, y, z)$ basis, the stress is calculated via $\bar{\sigma}=\overline{\bar{R}}^{\top} \times$ $\overline{\sigma^{\prime}} \times \overline{\bar{R}}$ to be

$$
\overline{\sigma_{m}}=\frac{\sigma}{2}\left[\begin{array}{l}
1 \\
1 \\
0 \\
0 \\
0 \\
1
\end{array}\right]
$$

where $\sigma$ is the magnitude of the stress applied. We can now apply Eq. (A4) to determine $\Delta \bar{B}$ :

$$
\Delta \overline{B_{m}}=\frac{\sigma}{2}\left[\begin{array}{c}
q_{11}+q_{12} \\
q_{11}+q_{12} \\
2 q_{12} \\
0 \\
0 \\
q_{44}
\end{array}\right]
$$

Since, however, we want to probe the birefringence in the $\left(x^{\prime}, y^{\prime}, z^{\prime}\right)$ basis, we apply the inverse rotation transformation $(\theta=\pi / 4)$ to determine $\Delta \overline{B^{\prime}}$ :

$$
\left[\begin{array}{c}
\Delta B_{1}^{\prime} \\
\Delta B_{2}^{\prime} \\
\Delta B_{3}^{\prime} \\
\Delta B_{4}^{\prime} \\
\Delta B_{5}^{\prime} \\
\Delta B_{6}^{\prime}
\end{array}\right]=\left[\begin{array}{c}
\Delta B_{x^{\prime} x^{\prime}}^{\prime} \\
\Delta B_{y^{\prime} y^{\prime}}^{\prime} \\
\Delta B_{z^{\prime} z^{\prime}}^{\prime} \\
\Delta B_{y^{\prime} z^{\prime}}^{\prime} \\
\Delta B_{x^{\prime} z^{\prime}}^{\prime} \\
\Delta B_{x^{\prime} y^{\prime}}^{\prime}
\end{array}\right]=\frac{\sigma}{2}\left[\begin{array}{c}
q_{11}+q_{12}+q_{44} \\
q_{11}+q_{12}-q_{44} \\
2 q_{12} \\
0 \\
0 \\
0
\end{array}\right],
$$

from which follows [using Eq. (A3)] a change in refractive index

$$
\left[\begin{array}{c}
\Delta n_{x^{\prime} x^{\prime}} \\
\Delta n_{y^{\prime} y^{\prime}} \\
\Delta n_{z^{\prime} z^{\prime}} \\
\Delta n_{y^{\prime} z^{\prime}} \\
\Delta n_{x^{\prime} z^{\prime}} \\
\Delta n_{x^{\prime} y^{\prime}}
\end{array}\right]=\left[\begin{array}{c}
n_{x^{\prime} x^{\prime}}-n_{0} \\
n_{y^{\prime} y^{\prime}}-n_{0} \\
n_{z^{\prime} z^{\prime}}-n_{0} \\
n_{y^{\prime} z^{\prime}}-n_{0} \\
n_{x^{\prime} z^{\prime}}-n_{0} \\
n_{x^{\prime} y^{\prime}}-n_{0}
\end{array}\right]=-\frac{\sigma}{4} n_{0}^{3}\left[\begin{array}{c}
q_{11}+q_{12}+q_{44} \\
q_{11}+q_{12}-q_{44} \\
2 q_{12} \\
0 \\
0 \\
0
\end{array}\right] .
$$

We are primarily interested in the birefringence between axes $\left(x^{\prime}, y^{\prime}\right)$, namely $\Delta n=\Delta n_{x^{\prime} x^{\prime}}-\Delta n_{y^{\prime} y^{\prime}}=n_{x^{\prime} x^{\prime}}-n_{y^{\prime} y^{\prime}}$. In the experiment, the stress is applied along $y^{\prime}$. In this case, $\overline{\sigma^{\prime}}=\left[\begin{array}{llllll}0 & 1 & 0 & 0 & 0 & 0\end{array}\right]^{\top}$, and $\Delta n=n_{x^{\prime} x^{\prime}}-n_{y^{\prime} y^{\prime}}=$ $\left(n_{0}^{3} / 2\right) \times q_{44} \times \sigma$, where $\sigma$ in this case has the inverse sign as in the case of stress applied along $x^{\prime}$, from which we obtain Eq. (2).

\section{Band-gap shift with uniaxial stress}

In order to calculate the excitonic emission shift as a result of uniaxial stress (along $x^{\prime}$ ) we assume that the shift is determined solely by the shift in the band gap of the host material, GaAs. We start with the Bir-Pikur expression [29] for the band-gap shift $\Delta E_{\text {gap }}$ with applied strain $\overline{\overline{\epsilon_{i j}}}$ in the usual basis $(x, y, z)$. The quantum dots themselves define the quantization axis, i.e., $z=[001]$. Assuming further that the valence state is of pure heavy-hole character, on account of the large heavy-hole-light-hole splitting,

$$
\Delta E_{\text {gap }}=a_{\Gamma} \operatorname{Tr}\left(\epsilon_{i j}\right)+\frac{b}{2}\left(2 \times \epsilon_{z z}-\epsilon_{x x}-\epsilon_{y y}\right),
$$

where $a_{\Gamma}$ and $b$ are the deformation potential coefficients, and $\operatorname{Tr}\left(\epsilon_{i j}\right)$ is the trace of the strain tensor $\overline{\overline{\epsilon_{i j}}}$.

We apply a stress, and thereby induce a strain. We use the strain-stress relation $\overline{\epsilon_{m}}=\overline{\overline{S_{m n}}} \times \overline{\sigma_{n}}$, where $\overline{\overline{S_{m n}}}$ is the compliance tensor, abbreviated in a similar way to Eq. (A4) on account of symmetry. We now know also the expression for a uniaxial stress along $x^{\prime}$ in the usual basis [Eq. (A6)]. 
The strain-stress relation reads

$$
\begin{aligned}
{\left[\begin{array}{c}
\epsilon_{x x} \\
\epsilon_{y y} \\
\epsilon_{z z} \\
\epsilon_{y z} \\
\epsilon_{x z} \\
\epsilon_{x y}
\end{array}\right]=\frac{\sigma}{2}\left[\begin{array}{cccccc}
S_{11} & S_{12} & S_{12} & 0 & 0 & 0 \\
S_{12} & S_{11} & S_{12} & 0 & 0 & 0 \\
S_{12} & S_{12} & S_{11} & 0 & 0 & 0 \\
0 & 0 & 0 & S_{44} & 0 & 0 \\
0 & 0 & 0 & 0 & S_{44} & 0 \\
0 & 0 & 0 & 0 & 0 & S_{44}
\end{array}\right]\left[\begin{array}{l}
1 \\
1 \\
0 \\
0 \\
0 \\
1
\end{array}\right] } \\
=\frac{\sigma}{2}\left[\begin{array}{c}
S_{11}+S_{12} \\
S_{11}+S_{12} \\
2 S_{12} \\
0 \\
0 \\
S_{44}
\end{array}\right] .
\end{aligned}
$$

Now, $\operatorname{Tr}\left(\epsilon_{m}\right)=\epsilon_{x x}+\epsilon_{y y}+\epsilon_{z z}=\left(S_{11}+2 \quad S_{12}\right) \quad \sigma \quad$ and $2 \epsilon_{z z}-\epsilon_{x x}-\epsilon_{y y}=\left(S_{12}-S_{11}\right) \sigma$. Transforming [26] $\overline{\overline{S_{m n}}}$ to the stiffness tensor $\overline{\overline{C_{m n}}}$, Eq. (A10) gives us the band-gap shift as a function of a stress along [110]:

$$
\delta E_{\mathrm{gap}} / \delta \sigma=\left(\frac{a_{\Gamma}}{C_{11}+2 C_{12}}-\frac{b}{2} \frac{1}{C_{11}-C_{12}}\right) .
$$

We use the following numerical values for GaAs from literature [30-32] for the computation: $a_{\Gamma}=-8.33 \mathrm{eV}$, $b=-2.00 \mathrm{eV}, C_{11}=122.3 \mathrm{GPa}$, and $C_{12}=57.1 \mathrm{GPa}$. We finally arrive at $\delta E_{\text {gap }} / \delta \sigma=-22.2 \mu \mathrm{eV} / \mathrm{MPa}$.

\section{Piezo-optical coefficients $q_{44}$ at $T=4 \mathrm{~K}$}

Data on the piezo-optical coefficient $q_{44}$ of $\mathrm{Al}_{x} \mathrm{Ga}_{1-x} \mathrm{As}$ alloys can be found for measurements $[28,33]$ at $T=298$ $\mathrm{K}$, and for GaAs at $T=77 \mathrm{~K}$ [33]. However, this data is not available at $T=4 \mathrm{~K}$. The dispersion of these coefficients is linked to the band gap of the particular material. In

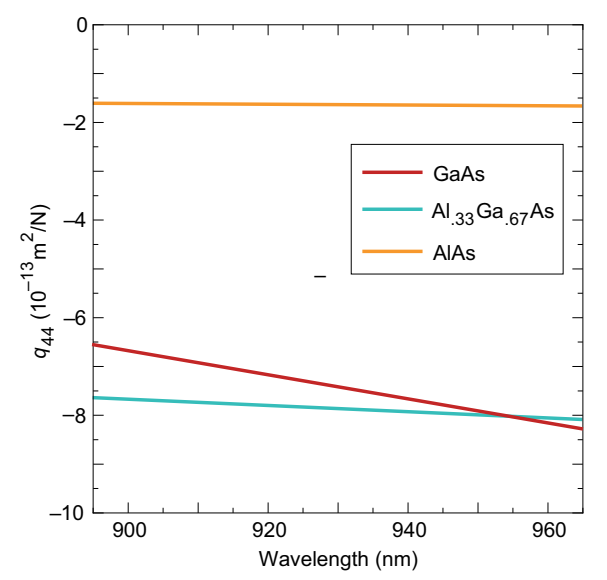

FIG. 5. Piezobirefringent coefficients $q_{44}$ at $T=4 \mathrm{~K}$ for GaAs, $\mathrm{Al}_{0.33} \mathrm{Ga}_{0.67} \mathrm{As}$, and $\mathrm{AlAs}$, estimated from their roomtemperature values by shifting the wavelength rigidly by an amount equal to the shift in band gap with temperature. particular, $q_{44}$ shows a resonance behavior at the band gap itself. As the band gap of these materials shifts with temperature, the $q_{44}$ coefficients are temperature dependent. It is therefore necessary to estimate the $q_{44}$ values at $T=4$ $\mathrm{K}$. We elaborate here the procedure.

Adachi [28] provides data - we extract the data from the plots with Webplotdigitizer [34] - on $\mathrm{Al}_{x} \mathrm{Ga}_{1-x} \mathrm{As}$ alloys, of particular relevance here the dispersion curve of the elasto-optic coefficients $p_{44}$, related to the piezobirefringent coefficients via $q_{44}=p_{44} \times S_{44}$. The room-temperature band-gap energies of the alloys of interest are also extracted $\left[E_{\text {gap }}(\mathrm{GaAs})=1.424 \mathrm{eV}\right.$, $E_{\text {gap }}\left(\mathrm{Al}_{0.33} \mathrm{Ga}_{0.67} \mathrm{As}\right)=1.8355 \mathrm{eV}, \quad E_{\text {gap }}(\mathrm{AlAs})=2.168$ $\mathrm{eV}]$. The optical properties of semiconductor crystals, such as the refractive index, are linked to the band-gap energy of the material [35]. The temperature dependence of the GaAs band gap can be described via $E_{\text {gap }}(T)=E_{\text {gap }}(0)-$ $5.405 \times 10^{-4} T^{2} /(T+204)$ [with $E_{\text {gap }}(T)$ in $\mathrm{eV}, T$ in $\mathrm{K}$ ] [36]. This equation was demonstrated to be valid also for $\mathrm{Al}_{x} \mathrm{Ga}_{1-x}$ As alloys [37].

From the room-temperature (298 K) band-gap energies, we can estimate the low-temperature band-gap energies of our materials, namely $E_{\text {gap }}(\mathrm{GaAs})=1.519 \mathrm{eV}$, $E_{\text {gap }}\left(\mathrm{Al}_{0.33} \mathrm{Ga}_{0.67} \mathrm{As}\right)=1.931 \mathrm{eV}, E_{\text {gap }}(\mathrm{AlAs})=2.263 \mathrm{eV}$, representing a shift in band-gap energy of $95 \mathrm{meV}$ for these materials. These shifts translate into a shift in wavelength of $\Delta \lambda=-54.45 \mathrm{~nm}, \Delta \lambda=-33.22 \mathrm{~nm}$, and $\Delta \lambda=$ $-24.01 \mathrm{~nm}$, respectively. We now estimate $q_{44}$ at $4 \mathrm{~K}$ for a particular wavelength $\lambda$ by rigidly shifting the curve of $q_{44}$ versus $\lambda$ at $298 \mathrm{~K}$ by $\Delta \lambda$. We confirm that this method functions well by comparing translated $T=298 \mathrm{~K}$ data [28] for $q_{44}$ to $T=77 \mathrm{~K}$ data [33] and verifying an overlap.

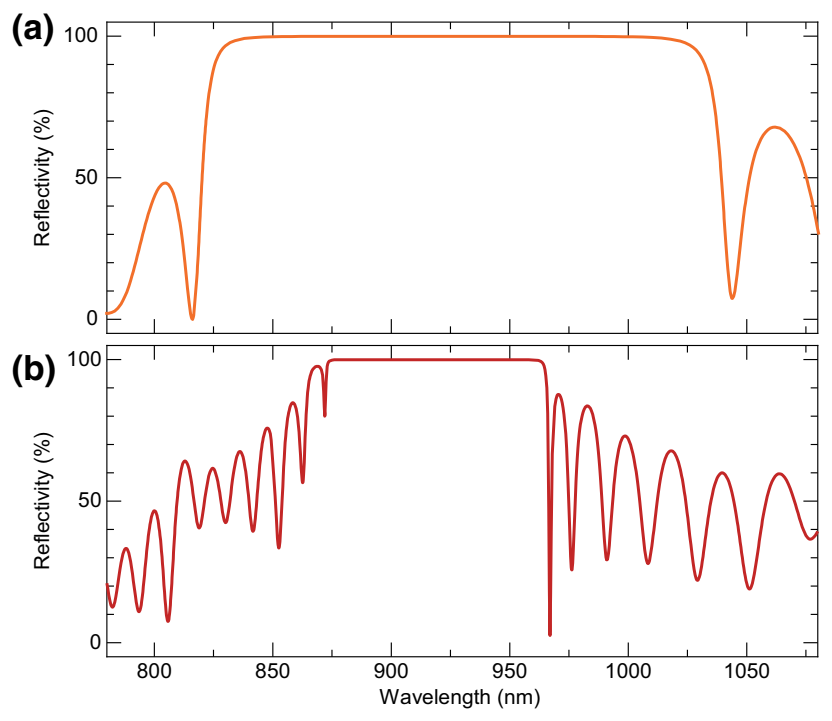

FIG. 6. Reflectivity spectra modeling the (a) top and (b) bottom mirrors composing the microcavity. The respective stopbands are centered at $\lambda=930.0 \mathrm{~nm}$ and $\lambda=918.7 \mathrm{~nm}$. 
Finally, we comment that the dispersion of $q_{44}$ of the semiconductor materials is rather small in the spectral band of interest, as exemplified in Fig. 5, such that we use their mean values in the model - we treat the small dispersion as a measure of the uncertainty in the parameters.

\section{Reflectivity spectra of cavity mirrors}

The models presented in this work are acquired using one-dimensional transfer-matrix simulation (Essential Macleod, Thin Film Center Inc.). For this, an appropriate model of the top and bottom mirrors composing the cavity is essential. In Fig. 6 the simulated reflectivity spectra of both mirrors is presented.

[1] H. Wang et al., Towards optimal single-photon sources from polarized microcavities, Nat. Photonics 13, 770 (2019).

[2] N. Tomm, A. Javadi, N. O. Antoniadis, D. Najer, M. C. Löbl, A. R. Korsch, R. Schott, S. R. Valentin, A. D. Wieck, A. Ludwig, and R. J. Warburton, A bright and fast source of coherent single photons, Nat. Nanotechnol. 16, 399 (2021).

[3] D. Najer, I. Söllner, P. Sekatski, V. Dolique, M. C. Löbl, D. Riedel, R. Schott, S. Starosielec, S. R. Valentin, A. D. Wieck, N. Sangouard, A. Ludwig, and R. J. Warburton, A gated quantum dot strongly coupled to an optical microcavity, Nature 575, 622 (2019).

[4] A. Dousse, J. Suffczyński, A. Beveratos, O. Krebs, A. Lemaître, I. Sagnes, J. Bloch, P. Voisin, and P. Senellart, Ultrabright source of entangled photon pairs, Nature 466, 217 (2010).

[5] J. Liu, R. Su, Y. Wei, B. Yao, S. F. C. Silva, Y. Yu, J. IlesSmith, K. Srinivasan, A. Rastelli, J. Li, and X. Wang, A solid-state source of strongly entangled photon pairs with high brightness and indistinguishability, Nat. Nanotechnol. 14, 586 (2019).

[6] I. Fushman, D. Englund, A. Faraon, N. Stoltz, P. Petroff, and J. Vučković, Controlled phase shifts with a single quantum dot, Science 320, 769 (2008).

[7] P. Lodahl, S. Mahmoodian, S. Stobbe, A. Rauschenbeutel, P. Schneeweiss, J. Volz, H. Pichler, and P. Zoller, Chiral quantum optics, Nature 541, 473 (2017).

[8] I. Söllner, S. Mahmoodian, S. L. Hansen, L. Midolo, A. Javadi, G. Kiršanske, T. Pregnolato, E.-E. Haitham, E. H. Lee, J. D. Song, S. Stobbe, and P. Lodahl, Deterministic photon-emitter coupling in chiral photonic circuits, Nat. Nanotechnol. 10, 775 (2015).

[9] M. P. van Exter, A. K. Jansen van Doorn, and J. P. Woerdman, Electro-optic effect and birefringence in semiconductor vertical-cavity lasers, Phys. Rev. A 56, 845 (1997).

[10] J. P. van der Ziel and A. C. Gossard, Absorption, refractive index, and birefringence of AlAs-GaAs monolayers, J. Appl. Phys. 48, 3018 (1977).

[11] J. E. Raynolds, Z. H. Levine, and J. W. Wilkins, Straininduced birefringence in GaAs, Phys. Rev. B 51, 10477 (1995).
[12] C. Bonato, D. Ding, J. Gudat, S. Thon, H. Kim, P. M. Petroff, M. P. van Exter, and D. Bouwmeester, Tuning micropillar cavity birefringence by laser induced surface defects, Appl. Phys. Lett. 95, 251104 (2009).

[13] J. A. Frey, H. J. Snijders, J. Norman, A. C. Gossard, J. E. Bowers, W. Löffler, and D. Bouwmeester, Electro-optic polarization tuning of microcavities with a single quantum dot, Opt. Lett. 43, 4280 (2018).

[14] S. Gerhardt, M. Moczała-Dusanowska, L. Dusanowski, T. Huber, S. Betzold, J. Martín-Sánchez, R. Trotta, A. Predojević, S. Höfling, and C. Schneider, Optomechanical tuning of the polarization properties of micropillar cavity systems with embedded quantum dots, Phys. Rev. B 101, 245308 (2020).

[15] S. Garcia, F. Ferri, K. Ott, J. Reichel, and R. Long, Dualwavelength fiber Fabry-Perot cavities with engineered birefringence, Opt. Express 26, 22249 (2018).

[16] K. Rechcińska, M. Król, R. Mazur, P. Morawiak, R. Mirek, K. Łempicka, W. Bardyszewski, M. Matuszewski, P. Kula, W. Piecek, P. G. Lagoudakis, B. Pietka, and J. Szczytko, Engineering spin-orbit synthetic hamiltonians in liquidcrystal optical cavities, Science 366, 727 (2019).

[17] S. Seidl, M. Kroner, A. Högele, K. Karrai, R. J. Warburton, A. Badolato, and P. M. Petroff, Effect of uniaxial stress on excitons in a self-assembled quantum dot, Appl. Phys. Lett. 88, 203113 (2006).

[18] L. Zhai, M. C. Löbl, J.-P. Jahn, Y. Huo, P. Treutlein, O. G. Schmidt, A. Rastelli, and R. J. Warburton, Large-range frequency tuning of a narrow-linewidth quantum emitter, Appl. Phys. Lett. 117, 083106 (2020).

[19] R. J. Barbour, P. A. Dalgarno, A. Curran, K. M. Nowak, H. J. Baker, D. R. Hall, N. G. Stoltz, P. M. Petroff, and R. J. Warburton, A tunable microcavity, J. Appl. Phys. 110, 053107 (2011).

[20] L. Greuter, S. Starosielec, D. Najer, A. Ludwig, L. Duempelmann, D. Rohner, and R. J. Warburton, A small mode volume tunable microcavity: Development and characterization, Appl. Phys. Lett. 105, 121105 (2014).

[21] D. Najer, N. Tomm, A. Javadi, A. R. Korsch, B. Petrak, D. Riedel, S. R. Valentin, R. Schott, A. D. Wieck, A. Ludwig, and R. J. Warburton, Suppression of Surface-Related Loss in a Gated Semiconductor Microcavity, Phys. Rev. Appl. 15, 044004 (2021)

[22] A. V. Kuhlmann, J. Houel, D. Brunner, A. Ludwig, D. Reuter, A. D. Wieck, and R. J. Warburton, A dark-field microscope for background-free detection of resonance fluorescence from single semiconductor quantum dots operating in a set-and-forget mode, Rev. Sci. Instrum. 84, 073905 (2013).

[23] R. N. Bhargava and M. I. Nathan, Stress dependence of photoluminescence in GaAs, Phys. Rev. 161, 695 (1967).

[24] F. H. Pollak and M. Cardona, Piezo-electroreflectance in Ge, GaAs, and Si, Phys. Rev. 172, 816 (1968).

[25] C. W. Higginbotham, M. Cardona, and F. H. Pollak, Intrinsic piezobirefringence of $\mathrm{Ge}, \mathrm{Si}$, and GaAs, Phys. Rev. 184, 821 (1969).

[26] J. F. Nye, Physical Properties of Crystals (Oxford University Press, Oxford, United Kingdom, 1957).

[27] Z. H. Levine, H. Zhong, S. Wei, D. C. Allan, and J. W. Wilkins, Strained silicon: A dielectric-response calculation, Phys. Rev. B 45, 4131 (1992). 
[28] S. Adachi, GaAs, AlAs, and $\mathrm{Al}_{x} \mathrm{Ga}_{1-x}$ As: Material parameters for use in research and device applications, J. Appl. Phys. 58, R1 (1985).

[29] G. L. Bir and G. E. Pikus, Symmetry and Strain-Induced Effects in Semiconductors (Wiley, New York, United States of America, 1974).

[30] Y. A. Burenkov, Y. M. Burdukov, S. Y. Davidov, and S. P. Nikaronov, Temperature dependences of the elastic constants of gallium arsenide, Sov. Phys. Solid State 15, 1175 (1973).

[31] C. G. Van de Walle, Band lineups and deformation potentials in the model-solid theory, Phys. Rev. B 39, 1871 (1989).

[32] Y. Sun, S. E. Thompson, and T. Nishida, Physics of strain effects in semiconductors and metal-oxide-semiconductor field-effect transistors, J. Appl. Phys. 101, 104503 (2007).

[33] A. Feldman and D. Horowitz, Dispersion of the piezobirefringence of GaAs, J. Appl. Phys. 39, 5597 (1968).

[34] A. Rohatgi, Webplotdigitizer: Version 4.3, (2020).

[35] N. M. Ravindra, P. Ganapathy, and J. Choi, Energy gaprefractive index relations in semiconductors - an overview, Infrared Phys. Technol. 50, 21 (2007).

[36] J. S. Blakemore, Semiconducting and other major properties of gallium arsenide, J. Appl. Phys. 53, R123 (1982).

[37] S. A. Lourenço, I. F. L. Dias, J. L. Duarte, E. Laureto, E. A. Meneses, J. R. Leite, and I. Mazzaro, Temperature dependence of optical transitions in AlGaAs, J. Appl. Phys. 89, 6159 (2001). 